This is a postprint version of the following published document:

A. Tapetado, D. S. Montero, C. Vázquez, and D. J. Webb, "WDM sensor network approach: Bridging the gap towards POF-based photonic sensing", in IEEE Sensors, Valencia (Spain), 2014, pp. 746-749.

Available in DOI: 10.1109/ICSENS.2014.6985107

(C) 2014. IEEE. Personal use of this material is permitted. Permission from IEEE must be obtained for all other uses, in any current or future media, including reprinting/republishing this material for advertising or promotional purposes, creating new collective works, for resale or redistribution to servers or lists, or reuse of any copyrighted component of this work in other works. 


\title{
WDM sensor network approach: bridging the gap towards POF-based photonic sensing
}

\author{
A. Tapetado*, D. S. Montero, C. Vázquez \\ Dpto. Tecnología Electrónica \\ Universidad Carlos III de Madrid \\ Leganés, Spain \\ *atapetad@ing.uc3m.es
}

\author{
D. J. Webb \\ Aston Institute of Photonics Technologies \\ Aston University \\ Birmingham, United Kingdom
}

\begin{abstract}
In this work a self-referenced technique for fiber-optic intensity sensors using virtual lock-in amplifiers is proposed and discussed. The topology is compatible with WDM networks so multiple remote sensors can simultaneously be interrogated. A hybrid approach combining both silica fiber Bragg gratings and polymer optical fiber Bragg gratings is analyzed. The feasibility of the proposed solution for potential medical environments and biomedical applications is shown and tested using a self-referenced configuration based on a power ratio parameter.
\end{abstract}

Keywords:

Self-referenced fiber-optic sensor network, polymer optical fiber Bragg grating (POFBG), biomedical applications.

\section{Introduction}

The number of fiber-optic sensors (FOS) for monitoring biomedical parameters is increasing and the ability to monitor patients requiring medical assistance is of great interest. Historically, the first application of optical fibers to the medical field enabled the illumination of internal organs during endoscopic procedures [1]. Nowadays, there are different medical areas where fiber-optic sensors hold enormous potential such as in clinical biomechanics, mainly if in-vivo applications are pursued $[2,3]$, as here there is a need to develop sensors for minimally invasive surgery procedures. Other optical-fiber advantages include small size, light weight, geometrical flexibility, chemical inertness, electric and thermal insulation, and immunity to electromagnetic interference. Potentially low-cost intensity-based optical biosensors made of tapered plastic optical fibers (POF) are developed in applications such as the detection of bacteria, yeast and erythrocytes by evaluating the output light power of the fiber containing the taper as a function of the refractive index of the surrounding medium [4]. It is clear that POFs are very attractive to be exploited in in-vivo sensing applications because they are made of a material inherently more biocompatible compare to silica fibers [5]. Anyhow, silica FBGs technology offers some advantages like self-referencing as the information is wavelength encoded and ease of multiplexing, facilitating distributed sensing. To give an example, multiple FBG sensors can be written in a single fiber and bonded at strategic locations for pressure mapping at different orthopedic joints either for patients' bed pressure profiling to continuously monitor their movements [6] and pressure exerted on human skin, such as for example in amputee sockets, shoe sensors, wheelchair seating-system sensors, hospital-bed monitoring sensors, rehabilitation robots and wearable exo-skeletons [7].
Nowadays polymer optical fiber Bragg gratings (POFBG) try to take advantage of polymer benefits such as larger elastic limit, higher maximum strain limit and larger temperature and humidity responses compared to silica while maintaining the benefits of FBG-based sensors [8, 9].

Nevertheless, limited effort has been directed towards synergizing biocompatible POF-based photonic sensing with the WDM interrogation method that allows multiplexing by the use of FBGs, with just a few exceptions [10]. The main underlying reason behind this lack of development is the mismatch between the optimum operating wavelength regions of POFs and the optical devices exploited for telecommunications purposes. As poly(methyl methacrylate) POF fibers has a high attenuation, around 1dB/cm @1550nm. Scenarios improving the power budget facilitate the integration of POF-based short-reach sensing networks near the patient and can minimize the great insertion loss and connection problems associated to POFBG-based fiber-optic sensors.

In this work, the feasibility of a hybrid silica-POF WDM network topology for addressing multiple self-referenced fiber-optic sensors using an output power ratio parameter is presented. The intention is to bridge the gap between the remote interrogation of multiple optical sensors and the advantages of using biocompatible POF-based sensors with low manufacturing cost, including those based on POFBGs.

\section{Theory}

Figure 1 shows the proposed hybrid self-referenced topology for remotely addressing intensity-based fiber-optic sensors. This configuration combines FBGs and POFBGs at the remote sensing points if a POF-based biocompatible sensing unit is required. It uses a self-referenced configuration that can be implemented either on a power ratio or on a phase parameter. Previous works consider a single delay with less flexibility [11], operate only on silica fiber networks and need 2 FBG for each sensing point [12-14] 


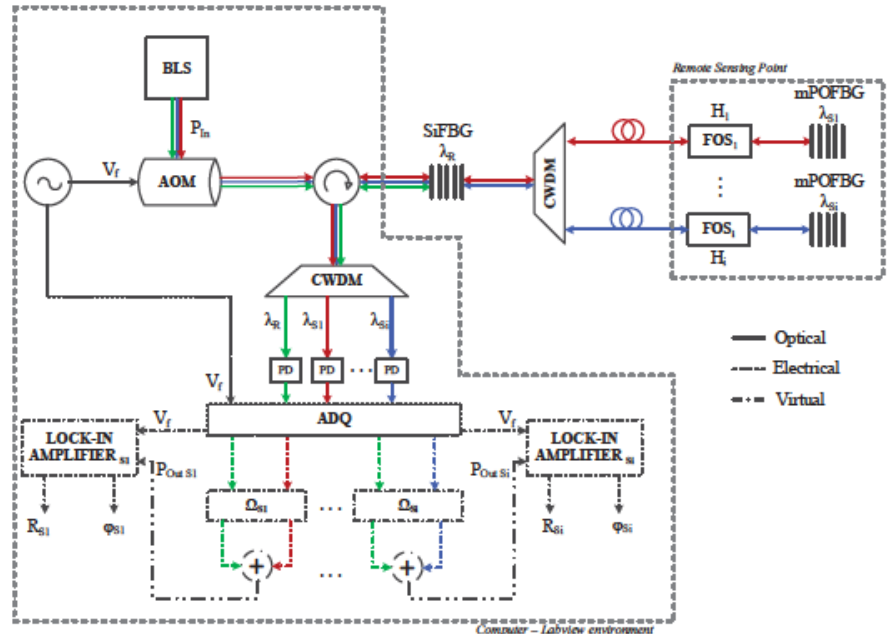

Figure 1.Point-to-point self-referenced topology for generic remote sensing points.

The centralized monitoring unit uses a modulated broadband light source (BLS) and some virtual lock-in amplifiers for the measurements. The BLS is externally modulated at a single frequency $f$ by an acousto-optic modulator (AOM). The modulated broadband signal is launched into the remote sensing points via a broadband circulator and a Coarse Wavelength-Division Multiplexer (CWDM). Each remote sensing point consists of a sensing POFBG placed after the fiber-optic sensor (FOS). A common silica FBG is located before the CWDM for reference purposes. The central wavelengths for the reference and sensing FBGs are $\lambda_{\mathbf{R}}$ and $\lambda_{\mathrm{Si}}$, respectively. The broadband optical circulator receives the reflected multiplexing signals from both reference and sensing channels. Sensor information is encoded within the latter. The optical signal is demultiplexed by a CWDM device and distributed to an array of photodetectors. At the reception stage, a data acquisition board $(\mathrm{ADQ})$ is used to convert the analog electrical signals to digital electrical signals. A digital phase-shift is applied to each modulated signal at both the reference and sensing wavelengths. Virtual lock-in amplifiers are used to interrogate all available sensor channels.

The transfer function for the complete sensor topology using the Z-Transform domain is given by:

$$
\frac{\mathrm{P}_{\text {Out i }}}{\mathrm{P}_{\text {In }}}=\alpha^{\prime} \cdot\left(1+\beta_{\mathrm{i}} \cdot \mathrm{z}^{-1}\right)
$$

with

$$
\begin{gathered}
\mathrm{z}=\mathrm{e}^{\left(-\mathrm{j} \cdot\left(\Omega_{\mathrm{Si}}-\Omega_{\mathrm{R}}\right)\right)} \\
\alpha^{\prime}=\mathrm{m}_{\mathrm{R}} \cdot \mathrm{r}\left(\lambda_{\mathrm{R}}\right) \cdot \mathrm{d}_{\mathrm{R}} \cdot \mathrm{e}^{\left(-\mathrm{j} \cdot \Omega_{\mathrm{R}}\right)} \\
\beta_{\mathrm{i}}=\frac{\mathrm{m}_{\mathrm{Si}} \cdot \mathrm{R}\left(\lambda_{\mathrm{Si}}\right) \cdot \mathrm{d}_{\mathrm{Si}} \cdot \mathrm{L}_{\mathrm{DEV}}}{\mathrm{m}_{\mathrm{R}} \cdot \mathrm{R}\left(\lambda_{\mathrm{R}}\right) \cdot \mathrm{d}_{\mathrm{R}}} \cdot \mathrm{H}_{\mathrm{i}}^{2}
\end{gathered}
$$

where $\Omega_{\mathrm{R}}$ and $\Omega_{\mathrm{Si}}$ are the phase shifts for the reference and each sensing signal. Parameters $m_{R}, R\left(\lambda_{R}\right)$ and $d_{R}$ are the modulation index, the reflectivity of the FBG and the photodetector response, respectively, at the reference wavelength, whereas $\mathrm{m}_{\mathrm{Si}}, \mathrm{R}\left(\lambda_{\mathrm{Si}}\right)$, and $\mathrm{d}_{\mathrm{Si}}$ are similar but for the sensing wavelength. $\mathrm{H}_{\mathrm{i}}$ is the sensor power loss modulation and appears two times due to the reflective operation of the sensing structure. Finally, $\mathrm{L}_{\mathrm{DEV}}$ is the insertion loss for the CWDM and POFBG.

Two measurement parameters can be defined for each remote sensing point, adapted from [11]: on one hand the parameter $\delta \mathrm{i}$, which is defined as the output ratio between the voltage values at the reception stage for different phase-shifts on the reference and sensing signals; on the other hand the output phase of the signal for different phase-shifts at the reception stage. The expressions for both measurement parameters are given by Eq. (4) and Eq. (5).

$$
\begin{gathered}
\delta_{\mathrm{i}}=\frac{\left.\mathrm{V}_{\mathrm{O}}\left(\mathrm{f}, \Omega_{\mathrm{Si}}\right)\right|_{\Omega_{\mathrm{R}}=0}}{\left.\mathrm{~V}_{\mathrm{O}}\left(\mathrm{f}, \Omega_{\mathrm{R}}\right)\right|_{\Omega_{\mathrm{i}}=0}}=\frac{\left[1+\frac{2 \cdot \beta_{\mathrm{i}}}{1+\beta_{\mathrm{i}}} \cos \left(\Omega_{\mathrm{Si}}\right)\right]^{1 / 2}}{\left[1+\frac{2 \cdot \beta_{\mathrm{i}}}{1+\beta_{\mathrm{i}}} \cos \left(-\Omega_{\mathrm{R}}\right)\right]^{1 / 2}} \\
\phi_{\mathrm{i}}=\operatorname{arctg}\left[\frac{-\left(\sin \Omega_{\mathrm{R}}+\beta_{\mathrm{i}} \cdot \sin \Omega_{\mathrm{Si}}\right)}{\left(\cos \Omega_{\mathrm{R}}+\beta_{\mathrm{i}} \cdot \cos \Omega_{\mathrm{Si}}\right)}\right]
\end{gathered}
$$

Both parameters $\delta \mathrm{i}$ and $\square \mathrm{i}$ are insensitive to power fluctuations outside the optical fiber link between the sensing and the reference Fiber Bragg gratings, and they are dependent on the sensor modulation $\left(\mathrm{H}_{\mathrm{i}}\right)$, thus performing a selfreference measurement parameter.

Depending on the selected self-reference parameter, 1 or 2 lock-in amplifiers are needed for each sensing channel, apart from the lock-in processing the reference channel.

\section{Measurements}

A 2-sensor network using the power ratio parameter was implemented to test the concept. A BLS modulated at $\mathrm{f}=1 \mathrm{kHz}$ by an acousto-optic modulator was employed to launch optical power into the configuration via a broadband circulator. One silica FBG was used for reference purposes, being placed after the broadband circulator and before the CWDM mux/demux. Its central wavelength and reflectivity were $\lambda_{R}=1550 \mathrm{~nm}$ and $49 \%$, respectively. A FBG in fewmoded microstructured Polymer Optical Fiber (mPOF) was used for each remote sensing point [8, 15]. Their central wavelengths were $\lambda_{\mathrm{s} 1}=1525.2 \mathrm{~nm}$ for $\mathrm{FOS}_{1}$ and $\lambda_{\mathrm{s} 2}=$ $1567.0 \mathrm{~nm}$ for $\mathrm{FOS}_{2}$, and their reflectivities were $27 \%$ and $36 \%$, respectively. The optical spectrum of the 2 -sensor network implemented is shown in Figure 2. 
Wavelength [nm]

$\begin{array}{llllllll}1510 & 1520 & 1530 & 1540 & 1550 & 1560 & 1570 & 1580\end{array}$

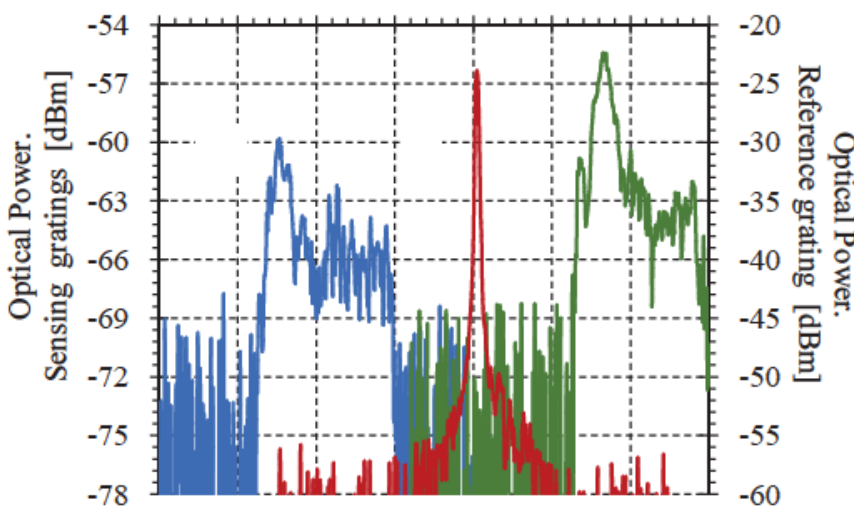

$\lambda \mathrm{S} 1=1525 \mathrm{~nm}$ (Sensing)

$\lambda \mathrm{S} 2=1567 \mathrm{~nm}$ (Sensing)

Figure 2. Optical spectrum in reflective operation for the $M P O F$ FBGs (sensing) and silica FBG (reference).

A single-mode Variable Optical Attenuator (VOA) was used to emulate the sensor response (FOS) and to calibrate the configuration. The two sensor loss modulations used to calibrate the configuration are shown in Figure 3.
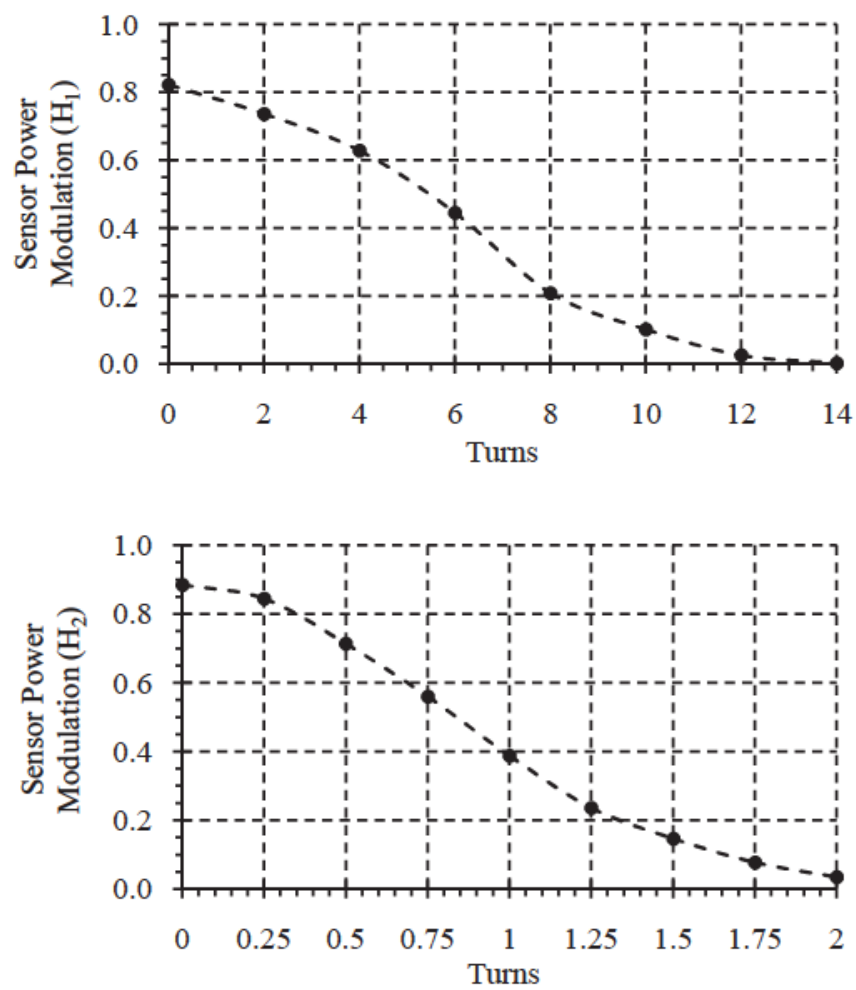

Figure 3. Calibration curves of the sensor loss modulation (a) $\mathrm{H}_{1}$ (b) $\mathrm{H}_{2}$ emulated by means of variable optical attenuators.

The reflected waves were demultiplexed by a CWDM and detected by three InGaAs photodetectors with $70 \mathrm{~dB}$ of gain. A low-cost $\mathrm{ADQ}$ was used to convert the electrical signals from the photodetectors to digital signals. The acquisition rate and the number of samples per channel was set to $16 \mathrm{kS} / \mathrm{s}$ and 8,000 samples, respectively. Computer software, based on Labview routines, was used to implement the phase-shifts and the lock-in amplifiers at the reception stage. Two virtual lockin amplifiers per sensor were used to obtain the selfreferencing parameter $\delta_{i}$, with $\mathrm{i}=1,2$. For the designed system, the resolution, in terms of output power variation, is $1.7 \cdot 10^{-2} \mathrm{~dB}$. The measurements of the self-referenced parameter showed repeatability errors of $0.1 \mathrm{mV}$. Its performance, i.e. linear behavior, maximum sensitivity, etc., is directly related to the digital phase-shifts applied at the reception stage. This behavior can be seen in Fig. 4 and Fig. 5, where the output ratio for different phase-shifts is illustrated. A less sensitivity behavior (slope) for lower phase-shift values and reference signal phase shifst closer to zero can be obtained. A good agreement between measurements (dot points) and simulation results using $\mathrm{Eq},(6)$ and a normalization procedure is achieved.

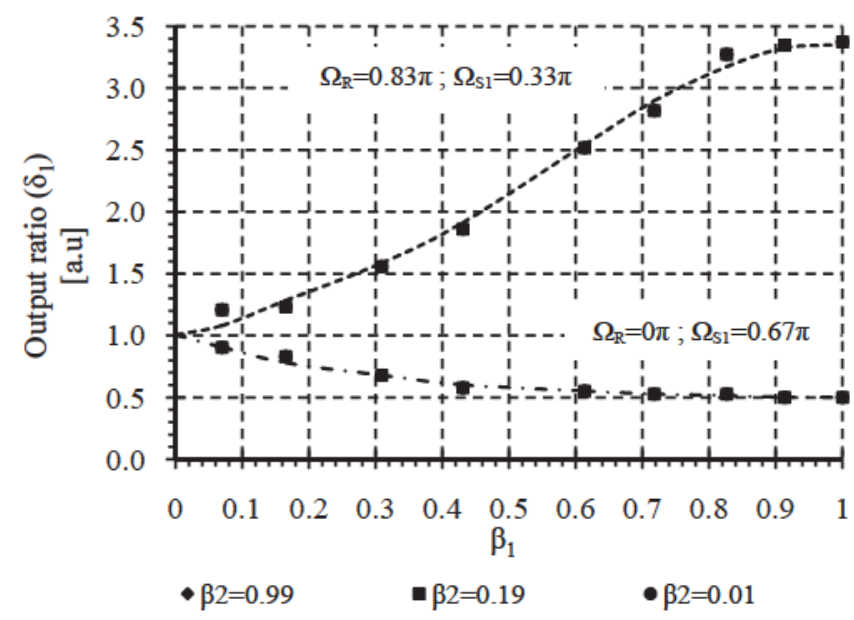

Figure 4. Measurement (rhombus, square and circle dots) and simulation (dashed and dash-dot line) results of the $\delta_{1}$ parameter versus $\beta_{1}\left(F O S_{1}\right)$ for different values of $\beta_{2}\left(\mathrm{FOS}_{2}\right)$

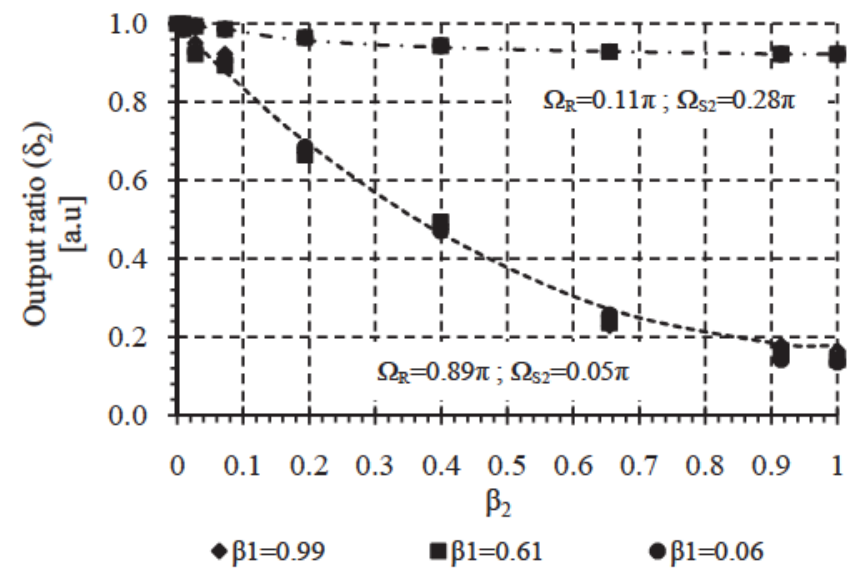

Figure 5. Measurement (rhombus, square and circle dots) and simulation (dashed and dash-dot line) results of the $\delta_{2}$ parameter versus $\beta_{2}\left(F_{O S}\right)$ for different values of $\beta_{1}\left(F_{O S}\right)$ 
Crosstalk analysis was carried out to measure the possible interference between adjacent channels during the measuring process. Several measurements of the self-referencing parameter $\delta_{1}\left(\mathrm{FOS}_{1}\right)$ were taken for different values of the sensor loss modulation $\beta_{2}\left(\mathrm{FOS}_{2}\right)$. Experimental results are shown in Figure 4. Similar results were obtained when monitoring $\delta_{2}$ when $\beta_{1}$ was changed, see Figure 5. In both cases no crosstalk was noticed, so both sensors can be interrogated simultaneously without mutual interference.

On the other hand, the self-reference property of the measurements' parameter was tested inducing power fluctuations in the modulated optical source through a variable optical attenuator. It can be seen at Figure 6 that there are no changes in the measurement parameter after inducing a power attenuation of $10 \mathrm{~dB}$.

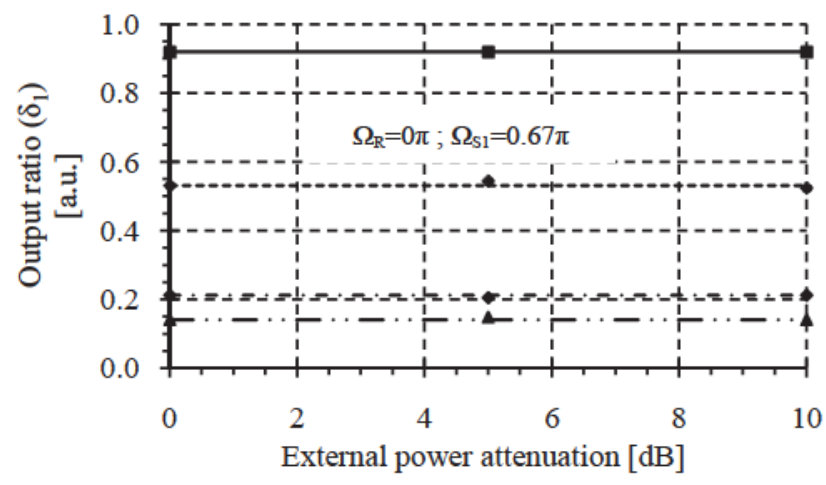

$\longrightarrow \beta 1=0.32-\cdots-\beta 1=0.54-\cdots-\beta 1=0.79-\cdots-\beta 1=0.92$

Figure 6. Self-reference test of $\delta_{1}$ versus power fluctuations up to $10 \mathrm{~dB}$ for different values of $\beta_{1}$.

\section{CONCLUSION}

A self-referenced sensor network topology with a central monitoring unit and performing a hybrid approach combining both silica FBGs and polymer FBGs (POFBGs) for simultaneous sensor interrogation is discussed. The proposed solution solves the inherent drawbacks of combining POFand silica-based optical components, being compatible with WDM networks. A central monitoring unit with virtual data processing is implemented. The WDM-based hybrid approach additionally allows extra power margins greater than $10 \mathrm{~dB}$ for synergizing POF short-reach sensing networks (units of meters) near the patient or improving the scalability of the architecture. The power margin achieved shows the feasibility of the proposed solution for potential medical environments and biomedical applications.

Proper selection of both virtual delays can lead to linear responses. The use of virtual instrumentation provides great flexibility and easy-reconfigurability. The self-referenced solution uses a low-cost 14-bit ADQ board which offers a system resolution of $1.7 \cdot 10^{-2} \mathrm{~dB}$ but can be improved using a lower noise photodetector and a ADQ with a higher resolution.

\section{Acknowledgment}

This work was supported by the Spanish Ministry of Economía y Competitividad under grant TEC2012-37983C03-02, BES-2010-033348 and EEBB-I-12-05434, and by ICT COST action TD1001. The authors wish to thanks Mr. Lutful Khan and Ms. Ada Abang for the help given to fabricate the MPOF Bragg gratings.

\section{References}

[1] K. Nishioka, and N. Yamashita, "Illumination optical system for an endoscope," Google Patents, 1983.

[2] J. Arkwright, N. Blenman, I Underhill et al., "In-vivo demonstration of a high resolution optical fiber manometry catheter for diagnosis of gastrointestinal motility disorders," Optics express, vol. 17, no. 6, pp. 4500-4508, 2009.

[3] D. J. Webb, S. Jones, L. Zhang et al., "First in-vivo trials of a fiber Bragg grating based temperature profiling system," Journal of biomedical optics, vol. 5, no. 1, pp. 45-50, 2000.

[4] C. Beres, F. V. B. de Nazaré, N. C. C. de Souza et al., "Tapered plastic optical fiber-based biosensor-Tests and application," Biosensors and Bioelectronics, vol. 30, no. 1, pp. 328-332, 2011.

[5] D. J. Webb, "Polymer photonic crystal fibre sensor applications." p. $77260 \mathrm{Q}$

[6] J. Hao, K. Tan, S. Tjin et al., "Design of a foot-pressure monitoring transducer for diabetic patients based on FBG sensors." pp. 23-24.

[7] G. T. Kanellos, G. Papaioannou, D. Tsiokos et al., "Two dimensional polymer-embedded quasi-distributed FBG pressure sensor for biomedical applications," Optics express, vol. 18, no. 1, pp. 179-186, 2010.

[8] H. Dobb, D. J. Webb, K. Kalli et al., "Continuous wave ultraviolet light-induced fiber Bragg gratings in few-and single-mode microstructured polymer optical fibers," Optics letters, vol. 30, no. 24, pp. 3296-3298, 2005

[9] J. Witt, M. Schukar, K. Krebber et al., "Heart rate sensor for integration into personal protective equipment." pp. 573-577.

[10] F. Berghmans, T. Geernaert, S. Sulejmani et al., "Photonic crystal fiber Bragg grating based sensors-opportunities for applications in healthcare." p. 831102.

[11] J. Montalvo, F. Araújo, L. Ferreira et al., "Electrical FIR filter with optical coefficients for self-referencing WDM intensity sensors," Photonics Technology Letters, IEEE, vol. 20, no. 1, pp. 45-47, 2008.

[12] D. Montero, C. Vázquez, J. Baptista et al., "Coarse WDM networking of self-referenced fiber-optic intensity sensors with reconfigurable characteristics," Optics express, vol. 18, no. 5, pp. 4396-4410, 2010.

[13] D. S. Montero, and C. Vázquez, "Remote Interrogation of WDM Fiber-Optic Intensity Sensors Deploying Delay Lines in the Virtual Domain," Sensors, vol. 13, no. 5, pp. 5870-5880, 2013.

[14] S.-J. Choi, Y.-C. Kim, M. Song et al., "A Self-Referencing Intensity-Based Fiber Optic Sensor with Multipoint Sensing Characteristics," Sensors, vol. 14, no. 7, pp. 12803-12815, 2014.

[15] G. Barton, M. A. van Eijkelenborg, G. Henry et al., "Fabrication of microstructured polymer optical fibres," Optical Fiber Technology, vol. 10 , no. 4, pp. 325-335, 2004 